\title{
Dynamic wake meandering model calibration using nacelle-mounted lidar systems
}

\author{
Inga Reinwardt ${ }^{1}$, Levin Schilling ${ }^{1}$, Peter Dalhoff $^{1}$, Dirk Steudel $^{2}$, and Michael Breuer ${ }^{3}$ \\ ${ }^{1}$ Dep. Mechanical Engineering \& Production, HAW Hamburg, Berliner Tor 21, 20099 Hamburg, Germany \\ ${ }^{2}$ Dep. Turbine Load Calculation, Nordex Energy GmbH, \\ Langenhorner Chaussee 600, 22419 Hamburg, Germany \\ ${ }^{3}$ Dep. of Fluid Mechanics, Helmut Schmidt University Hamburg, \\ Holstenhofweg 85, 22043 Hamburg, Germany
}

Correspondence: Inga Reinwardt (inga.reinwardt@ haw-hamburg.de)

Received: 19 November 2019 - Discussion started: 4 December 2019

Revised: 26 March 2020 - Accepted: 18 May 2020 - Published: 19 June 2020

\begin{abstract}
Light detection and ranging (lidar) systems have gained a great importance in today's wake characteristic measurements. The aim of this measurement campaign is to track the wake meandering and in a further step to validate the wind speed deficit in the meandering frame of reference (MFR) and in the fixed frame of reference using nacelle-mounted lidar measurements. Additionally, a comparison of the measured and the modeled wake degradation in the MFR was conducted. The simulations were done with two different versions of the dynamic wake meandering (DWM) model. These versions differ only in the description of the quasi-steady wake deficit. Based on the findings from the lidar measurements, the impact of the ambient turbulence intensity on the eddy viscosity definition in the quasi-steady deficit has been investigated and, subsequently, an improved correlation function has been determined, resulting in very good conformity between the new model and the measurements.
\end{abstract}

\section{Introduction}

Wake calculation of neighboring wind turbines is a key aspect of every wind farm development. The aim is to estimate both energy yield of the whole wind farm and loads on single turbines as accurately as possible. One of the main models for calculating the wake-induced turbulence in a wind farm is the so-called Frandsen model (see, for example, Frandsen, 2007). Previous measurement campaigns have shown that this model delivers conservative results for small turbine distances (Reinwardt et al., 2018; Gerke et al., 2018). This is particularly important for onshore wind farms in densely populated areas, where a high energy output per utilized area is crucial. In such cases, the usage of a more accurate description of the physical behavior of the wake, as defined in the dynamic wake meandering (DWM) model, seems appropriate. The DWM model is based on the assumption that the wake behaves as a passive tracer, which means the wake itself is deflected in the vertical and horizontal directions (Larsen et al., 2008b). The combination of this deflection and the shape of the wind speed deficit leads to an increased turbulence at a fixed position downstream. This plays an eminent role in the loads of a turbine located downstream of another turbine (Larsen et al., 2013). Therefore, a precise description of the meandering itself and the wind speed deficit in the meandering frame of reference (MFR) as well as a detailed validation of the wind speed deficit definition are fundamental.

Lidar systems are highly suitable for wake validation purposes. In particular, the so-called scanning lidar systems offer great potential for detailed wake analysis. These lidars are capable of scanning a three-dimensional wind field, so that the line-of-sight (LOS) wind speed can be measured subsequently at different positions in the wake, thus enabling the detection of the wake meandering as well as the shape of the wind speed deficit in the MFR. That is the reason why such a device is used in the measurement campaign outlined here. Several different measurement campaigns with ground-based and nacelle-mounted lidar systems have already been carried 
out in the last years, some of them even with the purpose of tracking wake meandering and validation of wake models.

In Bingöl et al. (2010) the horizontal meandering has been examined with a nacelle-installed continuous-wave $(\mathrm{CW})$ lidar. The campaign confirms the passive tracer assumption, which is essential for the definition of the meandering in the DWM model. Furthermore, the wind speed deficit in the MFR has been investigated for some distances. Due to the fact that the $\mathrm{CW}$ lidar can not measure simultaneously in different downstream distances, the beam has been focused successively to different downstream distances. In Trujillo et al. (2011) the analysis has been extended to a two-dimensional scan. The measured wind speed deficit in the MFR has been compared to the Ainslie wake model (Ainslie, 1988), which constitutes the basis of the deficit's definition in the DWM model.

Additionally, in Machefaux et al. (2013) a comparison of measured lateral wake meandering based on pulsed scanning lidar measurements has been presented. Special attention is paid to the advection velocity of the wake, which is estimated with measured and low-pass-filtered wind directions at the met mast (based on the assumptions of the DWM model) and the wake displacement at certain downstream distances. The analysis shows that the advection velocity calculated by the Jensen model is in relatively good agreement. Finally, the study compares the measured expansion of the wake in the fixed frame of reference (FFR) to computational fluid dynamics (CFD) simulations and simple analytical engineering models. The wake expansion calculated by simple analytical engineering models is well in line with lidar measurements and CFD simulations, but it also depicts potential for further improvements, which is why a new empirical model for single-wake expansion is proposed in Machefaux et al. (2015). In Machefaux et al. (2016) a measurement campaign is presented that involves three nacelle-mounted CW scanning lidar devices. The investigation includes a spectral analysis of the wake meandering, a comparison of the measurements to the assumptions in the DWM model, and a comparison of the wind speed deficit profile in a merged wake situation to CFD simulations.

It should be noted that the references listed here are only the most essential, on which the present measurement campaign builds. Several campaigns including either lidar systems or meandering observations as well as wake model validations have been conducted in the past. The outlined analysis transfers some of the procedures of tracking the wake meandering to measurement results from an onshore wind farm with small turbine distances. Particular focus is put on the investigation of the wind speed deficit's shape in the MFR and the degradation of the wind speed deficit in the downstream direction. The latter can be captured very well with the used nacelle-mounted pulsed scanning lidar systems due to the fact that it measures simultaneously in different downstream distances. Thus, a detailed comparison of the predicted degradation of the wind speed deficit between the DWM model and the measurement results is possible. Furthermore, the collected lidar measurements are used to recalibrate the DWM model, which enables a more precise modeling of the wake degradation. As a consequence, the calculation of loads and energy yield of the wind farm can be improved.

The remaining document is arranged as follows: in Sect. 2, the investigated wind farm and the installed measurement equipment are described in detail. Afterwards, in Sect. 3, an explanation of the data processing and filtering of the measurement results is given. Sections 4, 5, and 6 focus on the description of the theoretical background, and a hands-on implementation of the DWM model is introduced. Based on the outlined measurement results, a recalibration of the defined degradation of the wind speed deficit in the DWM model is proposed in Sect. 6. A summary of the measurement results can be found in Sect. 7, and a comparison to the original DWM model as well as the recalibrated version is presented in Sect. 8. Finally, all findings are concluded in Sect. 9.

\section{Wind farm}

The investigated onshore wind farm (Fig. 1) located in the southeast of Hamburg (Germany) consists of five closely spaced Nordex turbines (one N117 $3 \mathrm{MW}$ turbine and four N117 2.4 MW turbines) and an IEC-compliant $120 \mathrm{~m}$ met mast, which is situated two rotor diameters $(D=117 \mathrm{~m})$ ahead of the wind farm in the main wind direction (westsouthwest). It is equipped with 11 anemometers, two of which are ultrasonic devices; three wind vanes; two temperature sensors; two thermohygrometers; and two barometers. The sensors are distributed along the whole met mast, but at least one of each is mounted in the upper $8 \mathrm{~m}$ (see Fig. 2). The thrust as well as the power coefficient curves for both wind turbines are illustrated in Fig. 3. There are no other turbines in the immediate vicinity and the terrain is mostly flat. Only at further distances (more than $1 \mathrm{~km}$ ) is the terrain slightly hilly (approx. $40 \mathrm{~m}$ ). Two turbine nacelles are equipped with a pulsed scanning lidar system (Galion G4000). The wind farm layout with all installed measurement devices is shown in Fig. 1 (the displayed load measurements are not in the scope of this paper, but will be introduced in future publications). One lidar system is installed on top of the nacelle of WTG 2 (N117 2.4 MW), facing backwards. The second lidar system is installed inside the nacelle of WTG 1 (N117 $3 \mathrm{MW}$ ) and measures through a hole in the rear wall. In this case, mounting the device on top of the nacelle is not possible, as the area is occupied by a recuperator. The positions of both devices are displayed in Fig. 2. Even though the setup reduces the field of vision, the measurement campaign described in this paper is not influenced by this restriction. On the plus side, the lidar system is not exposed to weather. Finally, nacelle-mounted differential GPS systems help track 
the nacelle's precise position as well as yaw movements with a centimeter range accuracy.

\section{Data filtering and processing}

The lidar data are filtered in accordance with the wind direction, so that lidar data without free inflow of the wakegenerating turbine as well as lidar measurements in the induction zone of another turbine are rejected. This leads to the remaining wind direction sectors listed in Table 1 . The remaining sectors are relatively small, especially for the lidar on WTG 2, which reduces the amount of usable measurement data drastically. Additionally, the measured lidar data are sorted into turbulence intensity bins for the further validation and recalibration of the DWM model. The ambient conditions are determined by $10 \mathrm{~min}$ time series statistics from the met mast; hence only measurement results with free inflow at the met mast are useable. Only situations with normal power production of the wake-generating turbine are considered. The turbine operation mode is identified through the turbine's supervisory control and data acquisition (SCADA) system. The statistics of the $10 \mathrm{~min}$ time series are applied to identify the operational mode. Furthermore, the data have been analyzed according to yaw misalignments, so that no data with turbine misalignments greater than $6^{\circ}$ are considered in the analysis. The misalignment is determined by the GPS systems and the met mast wind direction. Moreover, the lidar data are filtered by the power intensity of the measurement results, which is closely related to the signal-to-noise ratio (SNR) of the measurements. Results with an intensity lower than 1.01 have been discarded. The pulse repetition rate of the lidar system is $15 \mathrm{kHz}$. The ray update rate is about $1 \mathrm{~Hz}$ (depending on the atmospheric conditions), so it averages over approximately 15000 pulses. The sample frequency is $100 \mathrm{MHz}$. Considering the speed of light, this delivers a point length of $1.5 \mathrm{~m}$. The range gate length is $30 \mathrm{~m}$; hence 20 points are used per range gate. The measurement time increases with the number of range gates, because the internal data processing time increases. Thus, to decrease the measurement time, the number of range gates has been limited, so that the farthest scan point is $750 \mathrm{~m}$ downstream. Additionally, the scanning time of each complete horizontal line scan is verified by the timestamp of each scan to ensure that the meandering can really be captured. In summary, this leads to the following filtering procedure for the measured lidar data.

1. Filter according to the wind direction determined by the met mast (free inflow at met mast and wind turbine and no induction zone from other turbines).

2. Filter according to the normal power production determined by the turbine's SCADA system.

3. Filter according to yaw misalignment.
Table 1. Considered wind direction sectors per wake-generating turbine in the measurement campaign. Wind direction sectors without free inflow of the met mast and the turbine as well as measurements in the induction zone of another turbine are omitted.

\begin{tabular}{lrr}
\hline & $\begin{array}{r}\text { Lower limit } \\
\left({ }^{\circ}\right)\end{array}$ & $\begin{array}{r}\text { Upper limit } \\
\left({ }^{\circ}\right)\end{array}$ \\
\hline WTG 1 & 160 & 190 \\
& 320 & 350 \\
\hline WTG 2 & 150 & 160 \\
& 240 & 250 \\
\hline
\end{tabular}

4. Filter according to the SNR of the lidar measurements.

5. Filter according to scan time.

6. Group all data sets in turbulence intensity bins with a bin width of $2 \%$.

Lidar systems measure the line-of-sight velocity. The wind speed in the downstream direction is then calculated from the lidar's LOS velocity and the geometric dependency of the position of the laser beam relative to the main flow direction as outlined in Machefaux et al. (2012). Thus, the horizontal wind speed is defined as

$U(t)=U_{\mathrm{LOS}} \cdot \frac{1}{\cos (\theta) \cdot \cos (\phi)}$,

where $\theta$ is the azimuth angle and $\phi$ the elevation angle of the lidar scan head. This seems to be a suitable approach for small scan opening angles like in the measurement campaign presented here. The biggest opening angle in the scan pattern is $20^{\circ}$. Nevertheless, if there is yaw misalignment, this could have an impact on the overall results. To decrease the uncertainties based on yaw misalignments, the measurement data have accordingly been filtered. The yaw misalignment has the biggest impact at the largest scan opening angle; i.e., a misalignment of $6^{\circ}$ at an opening angle of $20^{\circ}$ leads to an overestimation of the wind speed of less than $5 \%$.

\section{Wind speed deficit in HMFR calculation}

The meandering time series and the wake's horizontal displacement are determined with the help of a Gaussian fit. Trujillo et al. (2011) assume that the probability of the wake position in the vertical and horizontal directions is completely uncorrelated, so that the two-dimensional fitting function can be expressed as follows:

$f_{2 \mathrm{D}}=\frac{A_{2 \mathrm{D}}}{2 \pi \sigma_{y} \sigma_{z}} \exp \left[-\frac{1}{2}\left(\frac{\left(y_{i}-\mu_{y}\right)^{2}}{\sigma_{y}^{2}}+\frac{\left(z_{i}-\mu_{z}\right)^{2}}{\sigma_{z}^{2}}\right)\right]$,

where $\sigma_{y}$ and $\sigma_{z}$ are the standard deviations of the horizontal and vertical displacements $\mu_{y}$ and $\mu_{z}$, respectively. 


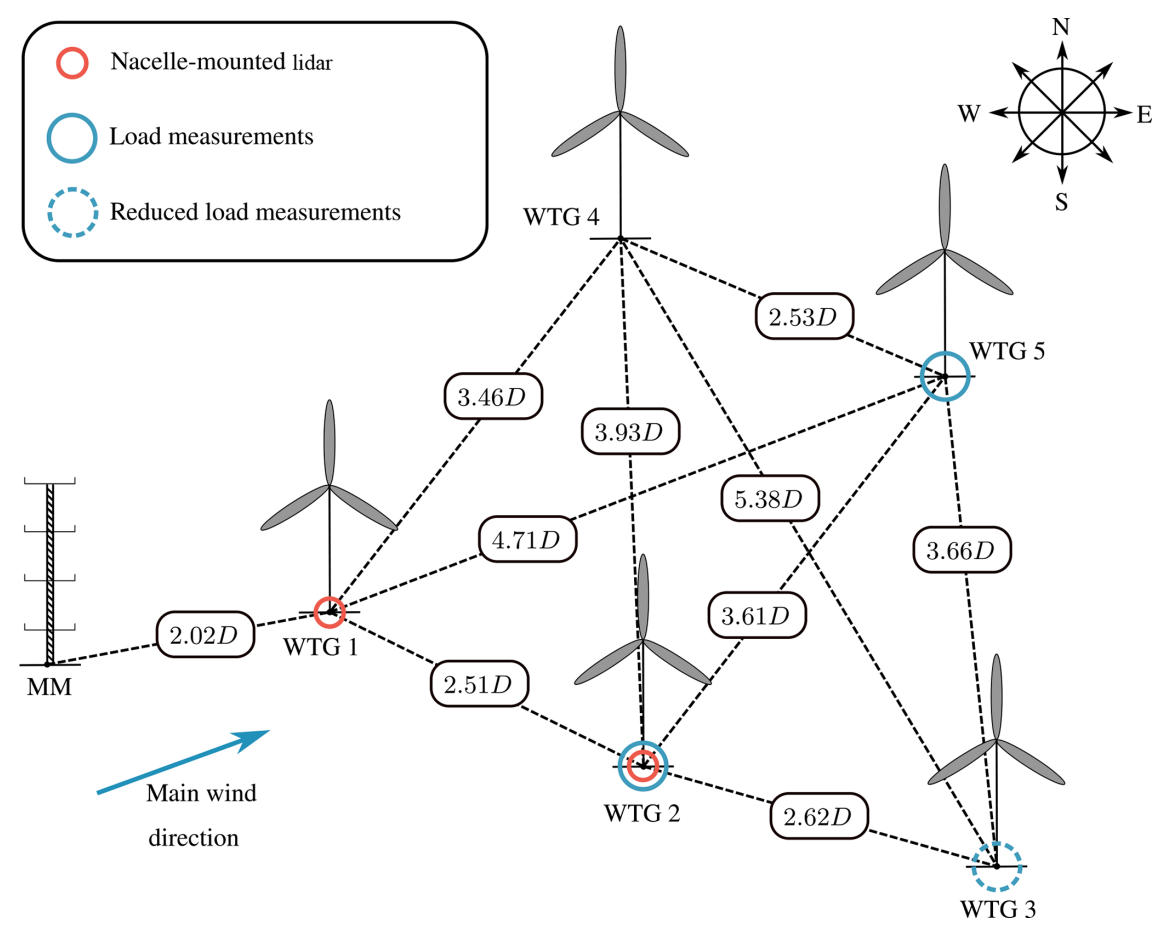

Figure 1. Wind farm layout with measurement equipment.

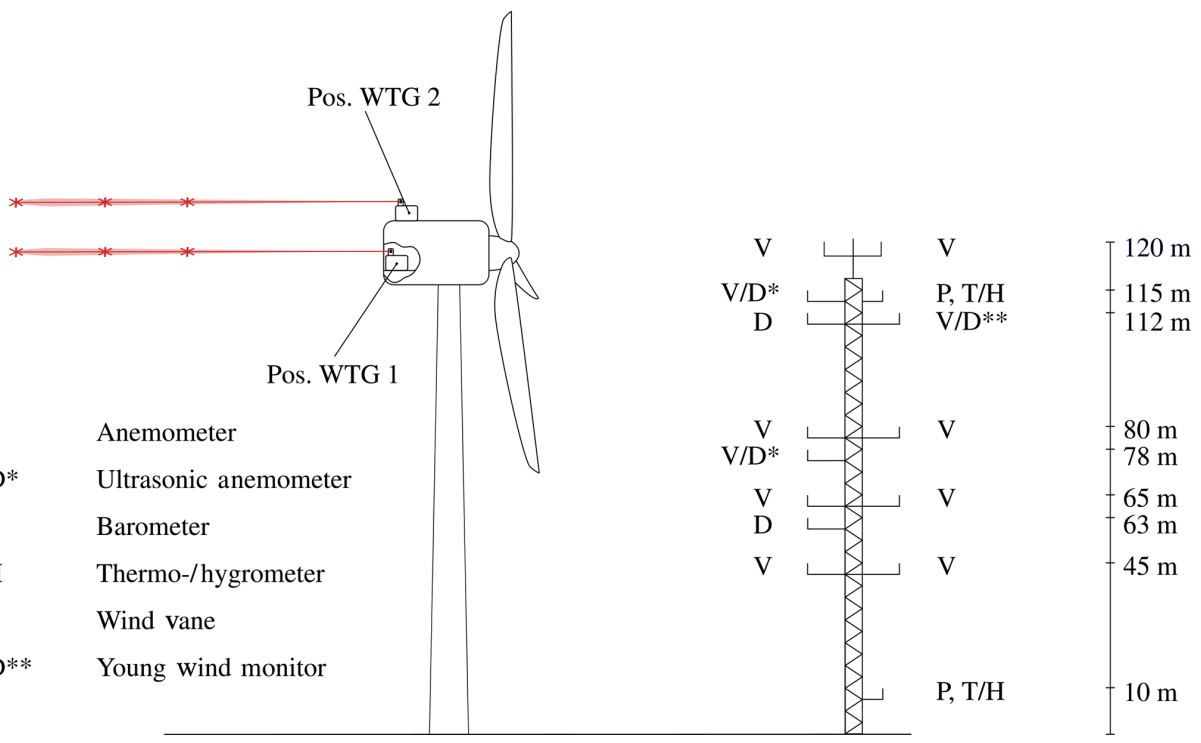

Figure 2. Met mast measurement equipment and lidar positions.

In the analysis presented here, only results from a horizontal line scan are analyzed, so that no vertical meandering is eliminated from the wind speed deficit, and the deficit's depth is less pronounced in comparison to the real MFR. To clarify that the vertical meandering is not eliminated in the present investigation, but included in the wind speed deficit, the abbreviation HMFR (horizontal meandering frame of reference) is introduced and henceforth used instead of MFR. A comparison of the wind speed deficit simulated with the
DWM model in the complete MFR and the HMFR is illustrated in Fig. 4. The simulations were carried out for a small downstream distance of $2.5 \mathrm{D}$ and a high turbulence intensity of $16 \%$. There are only small discrepancies around the center of the wake, which validates the present assumption.

Since the vertical meandering is neglected, the measurement results are fitted to a one-dimensional Gaussian curve 


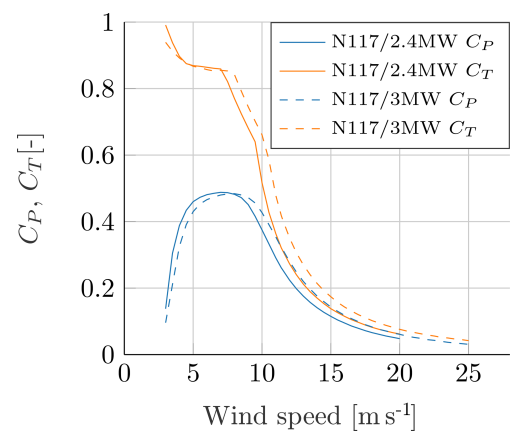

Figure 3. Power and thrust coefficients over wind speed for the N117 $3 \mathrm{MW}$ and the N117 2.4 MW turbines.

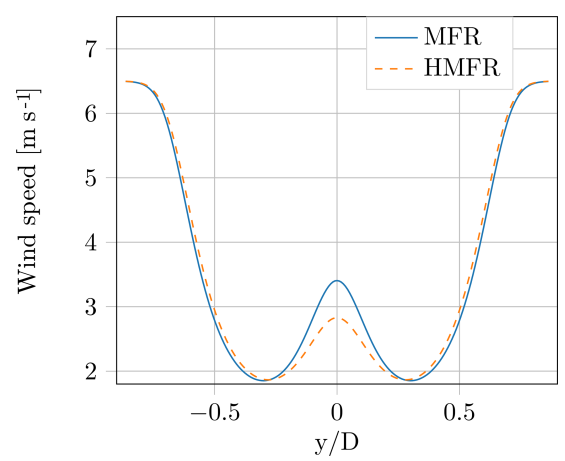

Figure 4. Wind speed deficit at a downstream distance of $2.5 \mathrm{D}$ and an ambient turbulence intensity of $16 \%$.

defined as follows:

$f_{1 \mathrm{D}}=\frac{A_{1 \mathrm{D}}}{\sqrt{2 \pi} \sigma_{y}} \exp \left(-\frac{1}{2} \frac{\left(y_{i}-\mu_{y}\right)^{2}}{\sigma_{y}^{2}}\right)$,

where $A_{1 \mathrm{D}}$ represents a scaling parameter. The measured wind speeds are fitted to the Gauss shape via a least-squares method. Thereby, only fitted horizontal displacements $\mu_{y}$ that are between -200 and $200 \mathrm{~m}$ are used for further validations of the mean wind speed in the HMFR. A horizontal displacement of more than $200 \mathrm{~m}$ cannot be represented by the Gauss fit due to a lack of measurement points. However, such an event is highly improbable (e.g., the DWM model predicts the wind speed deficit's probability at the horizontal position of $200 \mathrm{~m}$ to be $2 \times 10^{-22}$ for an ambient wind speed of $6.5 \mathrm{~m} \mathrm{~s}^{-1}$ and an ambient turbulence intensity of $8 \%$ ).

The entire method of calculating the wind speed deficit in the HMFR is illustrated in Fig. 5 and can be described as follows. The lidar system takes measurements from the nacelle of the turbine in the downstream direction, which deliver the wind speed deficit in the nacelle frame of reference or even in the FFR (see left side of Fig. 5) if the turbine is not moving (this is ensured by the GPS systems). A Gauss curve is then fitted into the scanned points as explained previously. It provides the horizontal displacement of the wake, so that each scan point can be transferred into the HMFR with the calcu- lated displacement (see middle diagrams in Fig. 5). The last step illustrated in the diagrams is the interpolation to a regular grid. These three steps are repeated for a certain number of scans $\mathrm{N}$ (e.g., approx. 37 for a $10 \mathrm{~min}$ time series). Finally, the mean value of all single measurement results in the HMFR is calculated. It should be noted that it is mandatory to interpolate to a regular grid. Otherwise it would not be possible to take the mean of all scans since the horizontal displacement differs at each instant of time, and, therefore, the measurement points are transmitted to a different location in the HMFR. After averaging, the plausibility of the results is inspected. If the calculated minimum mean wind speed in the HMFR is higher than the minimum mean wind speed in the FFR, it is assumed that the Gauss fit failed and the results are no longer considered. In theory, the wind speed deficit in the HMFR should be more pronounced than the measured one in the FFR, wherefore this fundamental plausibility check is added.

\section{Lidar simulation}

One of the most challenging parts of this specific measurement campaign is the low ray update rate of the lidar system, which is considerably smaller than in the previously introduced measurement campaigns (Bingöl et al., 2010; Trujillo et al., 2011). To ensure that the meandering as well as the wind speed deficit in the HMFR can be captured with the devices used, lidar and wind field simulations have been conducted in advance. The simulations incorporate lidar specifications (e.g., beam update rate and scan head angular velocity) and wind farm site conditions (ambient turbulence intensity and wind shear). The simulations assume perfect lidar measurements, where no probe volume averaging is considered and the lidar measures the horizontal wind speed directly. The wind field is simulated at halfway of the range gate. The simulated lidar "takes measurements" in a simulated wind field that is generated by the DWM model and includes wake effects as well as ambient turbulence. A detailed description of the model is given in Sect. 6. The inhouse code is written in Python. From these "measured" wind speeds the meandering is determined via Gaussian fits as previously explained and implemented in the real measurement campaign. Simulations are performed for different scan patterns, ambient conditions, and downstream distances to test the scan pattern, which for this one-dimensional scan consists of only 11 scan points scanned in a horizontal line from -20 to $20^{\circ}$ in $4^{\circ}$ steps. The "measurement" results of the simulated meandering time series are shown in Fig. 6a, whereas the corresponding wind speed deficit in the HMFR is presented in Fig. 6 b. The results are compared to the original meandering time series and the simulated wind speed deficit. The measured wind speed deficit in the simulated environment reproduces the simulated wind speed and its underlying meandering time series very well (the coefficient of 


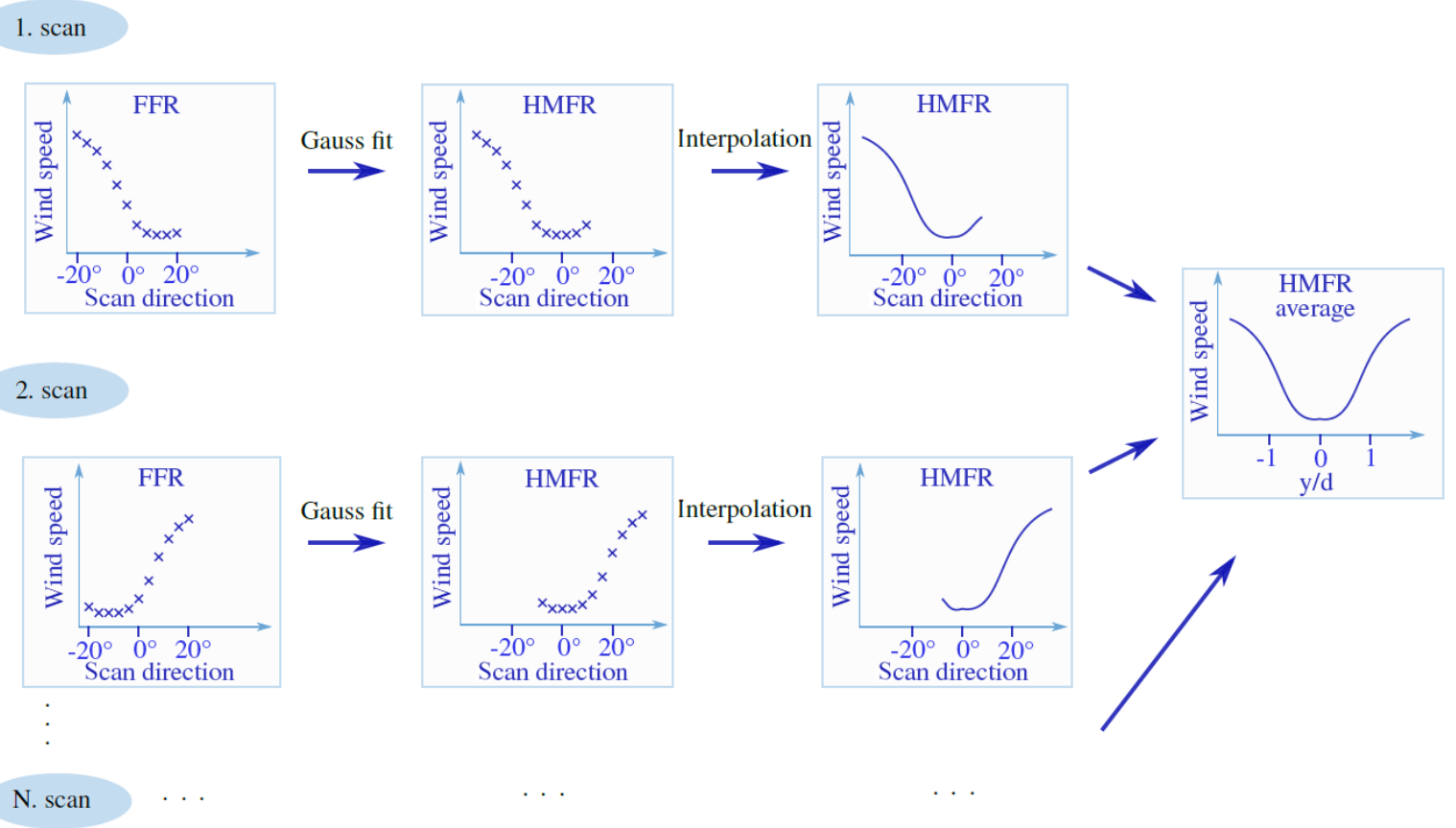

Figure 5. Method for the determination of the mean wind speed deficit in the HMFR.

determination $R^{2}$ is approximately 0.93 ). Although only 11 scan points are used for these plots, the curve of the wind speed deficit is very smooth. The reason for this behavior is the previously mentioned interpolation process. The distribution generated by the meandering process provides many scan points around the center of the wind speed deficit and only a few at the tails. Therefore, the influence of turbulence at the tails is much higher, leading to a somewhat coarse distribution at the boundaries of the deficit. It should also be noted that since this is a one-dimensional scan, the simulated lidar measures the wind speed deficit only horizontally, neglecting the wake's less dominant vertical movement. Whenever the wind speed deficit in the HMFR is mentioned in subsequent validations, it implies the neglect of eliminating the vertical meandering from the wind speed deficit, which has only a marginal impact on the shape of the wind speed deficit in the real MFR (see Fig. 4).

The lidar simulations indicate that the Gauss fit works more reliably under optimal operating conditions, i.e., at optimal tip speed ratio, when the wind speed deficit is most pronounced and the power coefficient $C_{\mathrm{p}}$ has its maximum (see Fig. 3). For the turbines examined, this applies to a range of 5 up to $8 \mathrm{~m} \mathrm{~s}^{-1}$, so that only measurement results with ambient wind speeds in this interval are analyzed.

\section{Dynamic wake meandering model}

The measured wind speed deficit in the HMFR is consecutively compared to the DWM model, which is based on the assumption that the wake behaves as a passive tracer in the turbulent wind field. Consequently, the movement of the passive structure, i.e., the wake deficit, is driven by large turbulence scales (Larsen et al., 2007, 2008b). The main components of the model are summarized in Fig. 7a. The model was built in house and independently of any commercial software in Python.

\subsection{Quasi-steady wake deficit}

One key point of the model is the quasi-steady wake deficit or rather the wind speed deficit in the MFR. In this study, two calculation methods for the quasi-steady wake deficit are compared with the lidar measurement results. A similar comparison of these models to met mast measurements in the FFR was published in Reinwardt et al. (2018). The quasi-steady wake deficit is defined in the MFR and consists of a formulation of the initial deficit emitted by the wakegenerating turbine and the expansion of the deficit downstream (Larsen et al., 2008a). The latter is calculated with the thin shear layer approximation of the Navier-Stokes equations in their axisymmetric form. This method is strongly related to the work of Ainslie (1988) and outlined in Larsen et al. (2007). The thin shear layer equations expressed by the wind speed in the axial and radial directions $U$ and $V_{\mathrm{r}}$, respectively, are defined by

$U \frac{\partial U}{\partial x}+V_{\mathrm{r}} \frac{\partial U}{\partial r}=\frac{1}{r} \frac{\partial}{\partial r}\left(v_{T} r \frac{\partial U}{\partial r}\right)$

and

$\frac{1}{r} \frac{\partial}{\partial r}\left(r V_{\mathrm{r}}\right)+\frac{\partial U}{\partial x}=0$. 


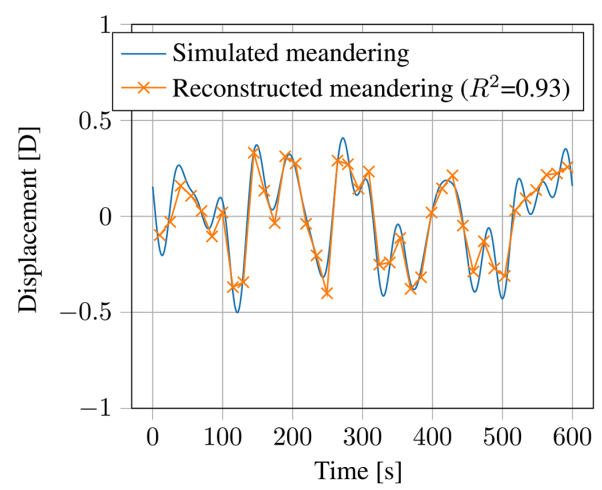

(a)

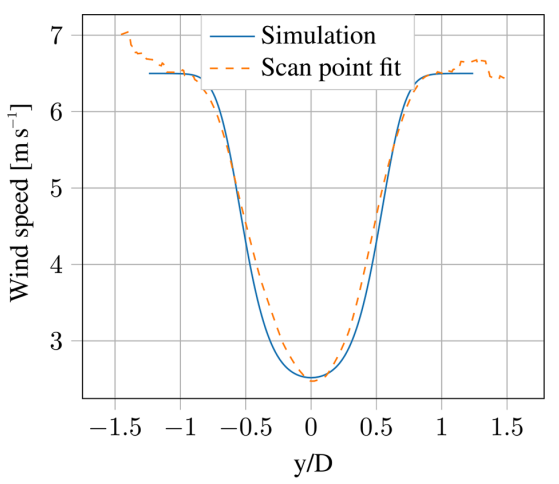

(b)

Figure 6. Simulated and simulated "measured" meandering time series (a) and wind speed deficit in the HMFR (b) at an ambient wind speed of $6.5 \mathrm{~m} \mathrm{~s}^{-1}$.

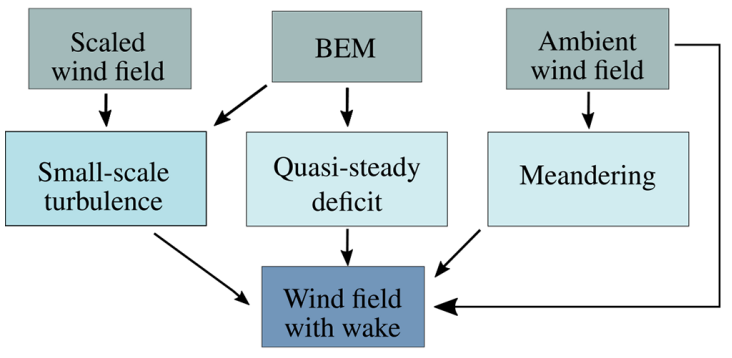

(a)

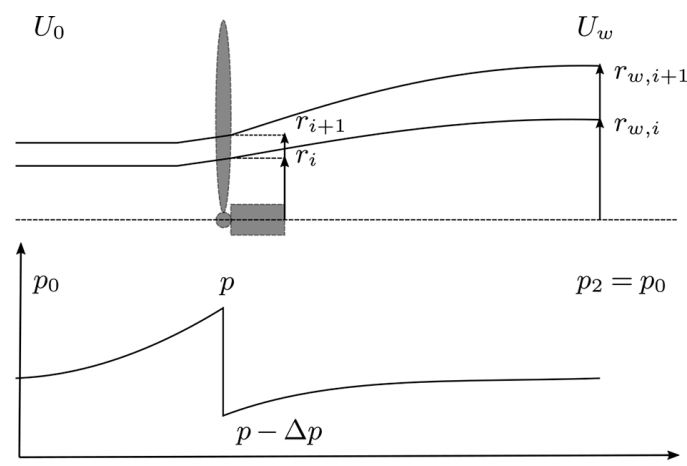

(b)

Figure 7. Components of the DWM model (a) (Reinwardt et al., 2018) and schematic illustration of the wake expansion in the DWM model (b) according to Madsen et al. (2010).

The first part of the quasi-steady wake deficit, the initial deficit, serves as a boundary condition when solving the equations. In both methods used to determine the quasisteady wake deficit, the initial deficit is based on the axial induction factor derived from the blade element momentum (BEM) theory. Pressure terms in the thin shear layer equations are neglected. The error that inherently comes with this assumption is accommodated by using the wind speed deficit two rotor diameters downstream (beginning of the far-wake area) as a boundary condition for the solution of the thin shear layer equations. The equations are solved directly from the rotor plane by a finite-difference method with a discretization in the axial and radial directions of $0.2 D$ and $0.0125 D$ combined with an eddy viscosity $\left(v_{T}\right)$ closure approach. The two methods that are compared with the lidar measurements only differ in the definition of the initial deficit and the eddy viscosity formulation.

\subsubsection{DWM-Egmond}

For the first method the following formulae are given to calculate the initial deficit. Hence, the boundary conditions for solving the thin shear layer equations are (Madsen et al., 2010)

$U_{\mathrm{w}}\left(\frac{r_{\mathrm{w}, i+1}+r_{\mathrm{w}, i}}{2}\right)=U_{0}\left(1-2 a_{i}\right)$

and

$r_{\mathrm{w}, i+1}=\sqrt{\frac{1-a_{i}}{1-2 a_{i}}\left(r_{i+1}^{2}-r_{i}^{2}\right)+r_{\mathrm{w}, i}^{2}} f_{\mathrm{w}}$

with

$f_{\mathrm{w}}=1-0.45 \bar{a}^{2}$,

where $\bar{a}$ represents the mean induction factor along all radial positions $i, r_{i}$ the rotor radius, and $r_{\mathrm{w}, i}$ the wake radius. The boundary condition of the radial velocity component is $V_{\mathrm{r}}=0$. The initial wake expansion and the corresponding radial positions as well as the pressure recovery in the downstream direction are illustrated in Fig. 7b. The eddy viscosity $v_{T}$ used in Eq. (4) is calculated in this first approach as fol- 
lows (Larsen et al., 2013):

$$
\begin{aligned}
\frac{\nu_{T}}{U_{0} R} & =k_{1} F_{1}(\tilde{x}) F_{\mathrm{amb}}(\tilde{x}) I_{0} \\
& +k_{2} F_{2}(\tilde{x}) \frac{R_{\mathrm{w}}(\tilde{x})}{R}\left(1-\frac{U_{\min }(\tilde{x})}{U_{0}}\right),
\end{aligned}
$$

with $k_{1}=0.1$ and $k_{2}=0.008$. The eddy viscosity is normalized by the ambient wind speed $U_{0}$ and the rotor radius $R$. The outlined definition consists of two terms. The first is related to the ambient turbulence intensity $I_{0}$, whereas the second depends on the shape of the wind speed deficit itself. The single terms are weighted with the factors $k_{1}$ and $k_{2}$. The filter functions $F_{1}$ and $F_{2}$ in Eq. (9) depending on $\tilde{x}$ (downstream distance normalized by the rotor radius) are defined by IEC 61400-1 (2019) as follows:

$F_{1}(\tilde{x})= \begin{cases}\left(\frac{\tilde{x}}{8}\right)^{3 / 2}-\frac{\sin \left(\frac{2 \pi \tilde{x}^{3 / 2}}{8^{3 / 2}}\right)}{2 \pi} & \text { for } 0 \leq \tilde{x}<8 \\ 1 & \text { for } \tilde{x} \geq 8\end{cases}$

and

$$
F_{2}(\tilde{x})= \begin{cases}0.0625 & \text { for } 0 \leq \tilde{x}<4 \\ 0.025 \tilde{x}-0.0375 & \text { for } 4 \leq \tilde{x}<12 \\ 0.00105(\tilde{x}-12)^{3} & \\ +0.025 \tilde{x}-0.0375 & \text { for } 12 \leq \tilde{x}<20 \\ 1 & \text { for } \tilde{x} \geq 20\end{cases}
$$

The filter function $F_{2}$ covers the lack of equilibrium between the velocity field and the rising turbulence in the beginning of the wake. $F_{1}$ is introduced to include the fact that the depth of the wind speed deficit increases in the near-wake area up to (2...3) $D$ downstream of the turbine until it attenuates again in the downstream direction (Madsen et al., 2010). The filter function as well as Eq. (8) are calibrated against actuator disc simulations at a downstream distance of $2 D$, the beginning of the far-wake area, where the wake is fully expanded (Madsen et al., 2010). A more detailed explanation of the nonlinear coupling function $F_{\text {amb }}$ is given in Sect. 6.3. This calculation method (Eqs. 6 to 11 ) is subsequently named "DWMEgmond" after the site, which is used for the calibration of the eddy viscosity in Larsen et al. (2013).

\subsubsection{DWM-Keck}

The second investigated method defines the initial deficit by the following equations (Keck, 2013):

$U_{\mathrm{w}}\left(r_{\mathrm{w}, i}\right)=U_{0}\left(1-\left(1+f_{u}\right) a_{i}\right)$

and

$r_{\mathrm{w}, i}=r_{i} \sqrt{\frac{1-\bar{a}}{1-\left(1+f_{R}\right) \bar{a}}}$,

with $f_{u}=1.1$ and $f_{R}=0.98$. The boundary condition of the radial velocity component is again $V_{\mathrm{r}}=0$. In Keck (2013) the final and recommended version of the model developed for the eddy viscosity is defined as follows:

$$
\begin{aligned}
v_{T} & =k_{1} F_{1}(\tilde{x}) u_{\mathrm{ABL} ; \lambda<2 D}^{*} l_{\mathrm{ABL} ; \lambda<2 D}^{*} \\
& +k_{2} F_{2}(\tilde{x}) \max \left(l^{* 2}\left|\frac{\partial U(\tilde{x})}{\partial r}\right|, l^{*}\left(1-U_{\min }(\tilde{x})\right)\right),
\end{aligned}
$$

with $k_{1}=0.578$ and $k_{2}=0.0178$ and the filter functions

$F_{1}= \begin{cases}\frac{\tilde{x}}{4} & \text { for } \tilde{x}<4 \\ 1 & \text { for } \tilde{x} \geq 4\end{cases}$

and

$F_{2}= \begin{cases}0.035 & \text { for } \tilde{x}<4 \\ 1-0.965 e^{-0.35(\tilde{x} / 2-2)} & \text { for } \tilde{x} \geq 4\end{cases}$

In contrast to the previously mentioned model (DWMEgmond), atmospheric stability is considered in this final model description. Equation (14) involves the velocity $u_{\mathrm{ABL} ; \lambda<2 D}^{*}$ and length scale $l_{\mathrm{ABL} ; \lambda<2 D}^{*}$ fractions of the ambient turbulence, which is related to the wake deficit evolution (eddies smaller than $2 D$ ). The velocity scale $u_{\mathrm{ABL} ; \lambda<2 D}^{*}$ is in addition to the ambient turbulence intensity $I_{0}$ related to the ratio of the Reynolds stresses (normal stress in the flow direction and the shear stress), which in turn are functions of the atmospheric stability. A detailed description of a method to introduce atmospheric stability in the DWM model can be found in Keck et al. (2014) and Keck (2013). In contrast to the final and recommended model in Keck (2013), atmospheric stability is not considered in this study, so that a previous model in Keck (2013) without consideration of atmospheric stability is used, and the numerical constants $k_{1}$ and $k_{2}$ in Eq. (17) are changed with respect to the first leastsquares recalibration in Keck (2013). Furthermore, according to Keck (2013) it can be assumed that the mixing length $l^{*}$ is equal to half of the wake width. This results in the following formulation of the eddy viscosity:

$$
\begin{aligned}
\frac{v_{T}}{U_{0} R}= & k_{1} F_{1}(\tilde{x}) I_{0}+k_{2} F_{2}(\tilde{x}) \max \left(\frac{R_{\mathrm{w}}(\tilde{x})^{2}}{R U_{0}}\left|\frac{\partial U(\tilde{x})}{\partial r}\right|,\right. \\
& \left.\frac{R_{\mathrm{w}}(\tilde{x})}{R}\left(1-\frac{U_{\min }(\tilde{x})}{U_{0}}\right)\right)
\end{aligned}
$$

with $k_{1}=0.0914$ and $k_{2}=0.0216$.

\subsection{Meandering of the wake}

The meandering of the wind speed deficit is calculated from the large turbulence scales of the ambient turbulent wind field. Thus, the vertical and horizontal movements are calculated from an ideal low-pass-filtered ambient wind field. The cutoff frequency of the low-pass filter is specified by the ambient wind speed and the rotor radius as (Larsen et al., 2013)

$$
f_{\mathrm{c}}=\frac{U_{0}}{4 R}
$$


The horizontal $y(t)$ and vertical $z(t)$ positions of the wind speed deficit are calculated based on the low-pass-filtered velocities in the horizontal and vertical directions according to the relations (Larsen et al., 2007)

$$
\frac{\mathrm{d} y(t)}{\mathrm{d} t}=v(t)
$$

and

$$
\frac{\mathrm{d} z(t)}{\mathrm{d} t}=w(t)
$$

where $v(t)$ and $w(t)$ are the fluctuating wind speeds at hub height. The ambient wind field, which is later on low-pass filtered, is generated in this work by a Kaimal spectrum and a coherence function (e.g., Veers, 1988). The temporal resolution of the generated wind field is $0.07 \mathrm{~s}$.

\subsection{Recalibration of the DWM model}

The wind speed deficit measured by the lidar systems is used to recalibrate the wake degradation downstream or to be more precise the eddy viscosity description. In Larsen et al. (2013) a recalibration was already achieved by introducing a nonlinear coupling function $F_{\mathrm{amb}}$ into the ambient turbulence intensity term of the eddy viscosity definition (see Eq. 9). Furthermore, a comparison between the measured and simulated power based on the DWM model was carried out. It shows that the wind speed deficit degradation is too low for lower turbulence intensities and moderate to high turbine distances in the model version from Madsen et al. (2010). For this reason, the downstream-distance-dependent function $F_{\text {amb }}$ was introduced into the eddy viscosity description in Larsen et al. (2013).

A similar behavior but even more pronounced can be seen in the results in Sect. 7. Following the approach of Larsen et al. (2013), a function based on a least-squares calibration with the acquired lidar measurements is developed. This function is incorporated into the normalized eddy viscosity description in Eq. (17), whereby it changes to

$$
\begin{aligned}
\frac{v_{T}}{U_{0} R}= & k_{1} F_{\mathrm{amb}}(\tilde{x}) F_{1}(\tilde{x}) I_{0} \\
& +k_{2} F_{2}(\tilde{x}) \max \left(\frac{R_{\mathrm{w}}(\tilde{x})^{2}}{R U_{0}}\left|\frac{\partial U(\tilde{x})}{\partial r}\right|,\right. \\
& \left.\frac{R_{\mathrm{W}}(\tilde{x})}{R}\left(1-\frac{U_{\min }(\tilde{x})}{U_{0}}\right)\right)
\end{aligned}
$$

with the constants $k_{1}=0.0924$ and $k_{2}=0.0216$ and the coupling function

$$
F_{\text {amb }}(\tilde{x})=a \tilde{x}^{-b}
$$

with $a=0.285$ and $b=0.742$. The parameters $a$ and $b$ are the results of the least-squares calibration. It should be noted that the constant $k_{1}$ was also slightly adjusted by the recalibration, in which the normalized eddy viscosity definition of Keck (2013) has been used. This derives from the fact that this model is already in good agreement with the measurement results in most turbulence intensity bins as demonstrated in Sect. 8 and also in Reinwardt et al. (2018).

\section{Measurement results}

The measurement campaign lasted from January to July 2019. Both lidar systems, introduced in Sect. 2, were used to collect the data. Results of the meandering time series over 10 min are exemplarily shown in Fig. 8a. The maximum displacement of the wake is about $0.5 \mathrm{D}$, which is equivalent to $58.5 \mathrm{~m}$. The results are derived from a $10 \mathrm{~min}$ time series with an ambient wind speed of $6.44 \mathrm{~m} \mathrm{~s}^{-1}$ and an ambient turbulence intensity of $11.7 \%$. Some of the met-mast-detected ambient conditions (wind speed $U_{0}$, turbulence intensity $I_{0}$, wind shear $\alpha$, and wind direction $\theta$ ) are given in the title of the figure. The corresponding mean wind speed deficit is illustrated in Fig. 8b. The wind speed decreases to less than $3 \mathrm{~m} \mathrm{~s}^{-1}$ in full-wake situations. As explained in Sect. 5, the tails of the curve are relatively coarse since fewer scan points were gathered. It can also be seen that the ambient wind speed is not even reached at the edges of the curve. The opening angle of the scan appears too small to capture the whole wake at this distance. Towards the left part of the wind speed deficit (at negative $y$ distances) a bigger part of the wake is captured. This arises from the fact that the horizontal displacement is more often positive than negative, and, therefore, more measurement results are collected towards the left part of the wind speed deficit curve.

The used lidar system is capable of measuring several range gates simultaneously in $30 \mathrm{~m}$ intervals. The results of all detected range gates for the data set presented in Fig. 8 are shown in Fig. 9a. The closest distance is $1.92 D$ downstream and the farthest is $6.28 \mathrm{D}$. The degradation of the wind speed deficit in the downstream direction is clearly identifiable. As for the single distance case (Fig. 8), for most range gates a bigger database is captured at the left part of the wind speed deficit, resulting in smoother curves. The presumption of a too small opening angle of the scan, as stated before, proves true. With increasing downstream distances the captured wind speed deficits get closer to integrity. A broader scan angle would result in more detailed wind speed deficits for close downstream distances at the expense of far distances, where the scan points might not capture enough points inside the deficit and thereby prevent a successful Gaussian fit. Furthermore, additional scan points at the edges can lead to a better representation of the deficit but would also increase the scan time. According to Eq. (18), the meandering is correlated to frequencies lower than approximately $0.028 \mathrm{~Hz}$ considering a wind speed of $6.5 \mathrm{~m} \mathrm{~s}^{-1}$ and a rotor diameter of $117 \mathrm{~m}$. This means that, considering the NyquistShannon sampling theorem, the scan time must be longer than half of the reciprocal of $0.028 \mathrm{~Hz}$, which results in a 


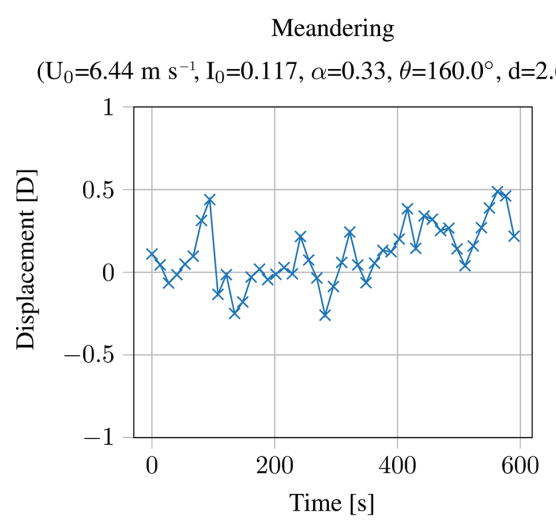

(a)
HMFR

$\left(\mathrm{U}_{0}=6.44 \mathrm{~m} \mathrm{~s}^{-1}, \mathrm{I}_{0}=0.117, \alpha=0.33, \theta=160.0^{\circ}, \mathrm{d}=2.69 \mathrm{D}\right)$

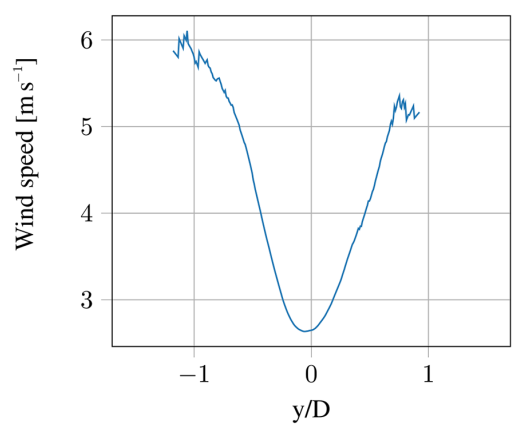

(b)

Figure 8. Meandering time series (a) and wind speed deficit in the HMFR (b) at 2.69D downstream of the turbine.

\section{HMFR}

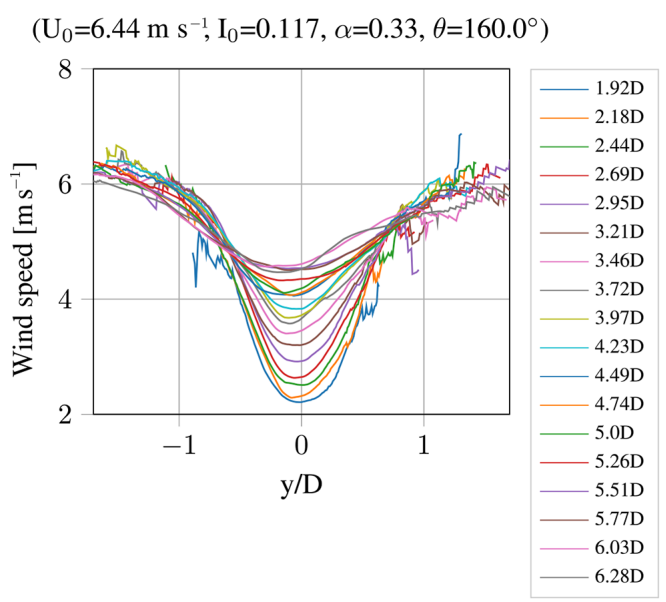

(a)

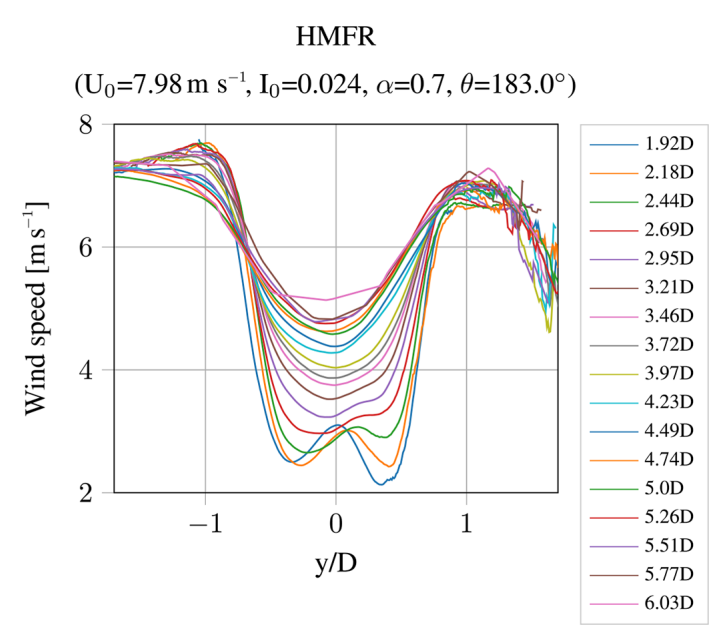

(b)

Figure 9. Wind speed deficit in the HMFR for an ambient turbulence intensity of $11.7 \%$ (a) and a turbulence intensity of $2.4 \%$ (b).

necessary scan time of less than $18 \mathrm{~s}$. The scan time for the current usage of 11 scan points is already at about $16 \mathrm{~s}$ (depending on the visibility conditions), which is close to the limit of $18 \mathrm{~s}$. Thus with an increased number of scan points it is no longer ensured that the meandering can be captured.

Figure $9 \mathrm{~b}$ illustrates the wind speed deficit in the HMFR measured under different ambient conditions. The corresponding meandering time series and wind speed deficit for this measured time series at $2.69 \mathrm{D}$ downstream are given in Fig. A1 in the Appendix. The wind shear is fairly high $(\alpha=$ $0.7)$ and the turbulence intensity is very low $\left(I_{0}=2.4 \%\right)$. Due to the low turbulence intensity it is still possible to see the " $\mathrm{W}$ " shape of the wind speed deficit at closer distances. The typical "W" shape is caused by the low axial induction in the area of the nacelle. Further downstream, the wake becomes more Gaussian shaped. At a horizontal distance of about $1.5 D$ from the wake center, the wind speed decreases. The reason is the wakes of other turbines in the wind farm.
The mean wind direction in this time series is $183^{\circ}$ and the measurements are taken from WTG 1 , so it could be the influence of the wake of either WTG 2 or WTG 4. The associated results of the mean wind speed deficit in the FFR are illustrated in Fig. 10. The curves in the FFR are less smooth than the wind speed deficit in the HMFR, simply because only 11 points are scanned and no interpolation is necessary when calculating the mean wind speed over the whole time series. Comparing Figs. 9 and 10, it becomes apparent that the wind speed deficit in the FFR is less pronounced. Furthermore, for the lower turbulence intensity the "W" shape of the wind speed is not visible, since it vanished due to the meandering.

Similar results as exemplarily shown in Figs. 9 and 10 have been collected for a multitude of different ambient conditions. The number of measured time series per turbulence intensity and wake-generating turbine, on which the lidar system is installed, is listed in Table 2 . The turbulence in- 
FFR

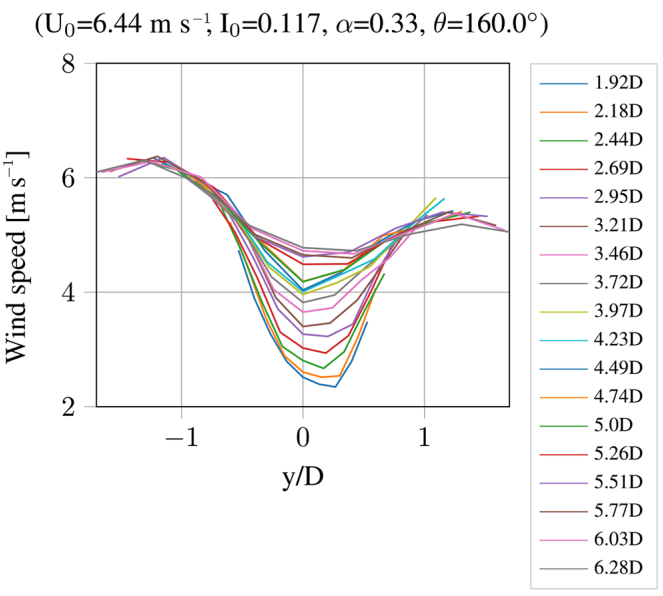

(a)
FFR

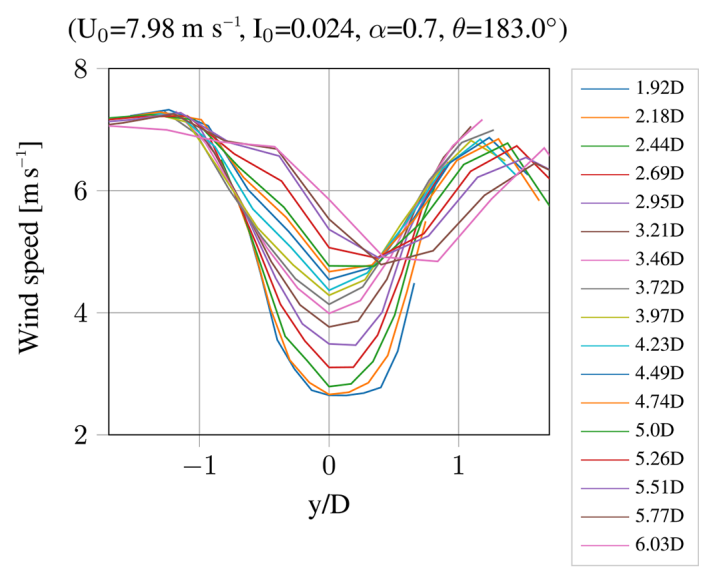

(b)

Figure 10. Wind speed deficit in the FFR for a turbulence intensity of $11.7 \%$ (a) and a turbulence intensity of $2.4 \%$ (b).

tensity is binned in $2^{\circ}$ steps. Column 1 of Table 2 specifies the mean values for each bin. Most of the measurement results are collected at low to moderate turbulence intensities $\left(I_{0}=4 \%-10 \%\right)$. Only a few results could be extracted at higher turbulence intensities. The results include time series with an ambient wind speed of 5 to $8 \mathrm{~m} \mathrm{~s}^{-1}$. In this range, both turbines operate under optimal and most efficient conditions, resulting in maximum energy output from the wind. The thrust coefficient is constant in this region (see Fig. 3). Therefore, the axial induction and the wind speed deficit normalized by the turbine's inflow wind speed are also expected to be constant for similar ambient conditions over this wind speed range. For the single turbulence intensity bins and both turbine types, simulations with different DWM models are carried out applying the same axial induction over the whole wind speed range. A scatterplot of the shear exponent and the ambient turbulence intensity determined by the met mast is given in Fig. 11. It includes all used data sets. At lower turbulence intensities, the shear spreads quite a lot, whereas towards higher turbulence intensities the shear decreases as expected.

Figure 12 summarizes all measured wind speed deficits in the HMFR. It demonstrates the mean value and the standard deviation of the mean for all captured turbulence bins plotted against the downstream distance. Each value is related to the minimum value of the wind speed deficit, which itself is normalized by the inflow wind speed. It should be noted that in some distances only one value satisfies the filtering and plausibility checks, whereby the error bar is omitted. Additionally, it is pointed out that the plotted values always refer to the minimum value of a wind speed curve and not necessarily to the velocity in the wake center. Therefore, no increase in the wind speed at low downstream distances on account of the "W" shape is visible. The wind speed deficit at the wake center plotted against the downstream distance is depicted in the
Table 2. Number of measured and considered data sets per turbulence intensity for the lidar systems on WTG 1 and WTG 2.

\begin{tabular}{lrr}
\hline$I_{0}(\%)$ & WTG 1 & WTG 2 \\
\hline 4 & 23 & 28 \\
6 & 8 & 11 \\
8 & 23 & 14 \\
10 & 11 & 9 \\
12 & 13 & 4 \\
14 & 0 & 0 \\
16 & 1 & 1 \\
18 & 1 & 2 \\
20 & 1 & 3 \\
22 & 0 & 2 \\
\hline
\end{tabular}

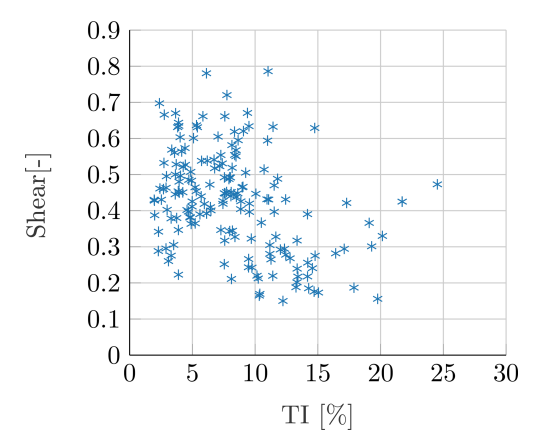

Figure 11. Shear exponent over the ambient turbulence intensity for all considered data sets.

next section in Fig. 15b and will be discussed further at this point. Figure 12 illustrates very well that the lowest degradation of the wind speed deficit occurs at the lowest turbulence intensity. Up to a turbulence intensity of $10 \%$, the degradation of the wind speed deficit continuously rises, leading to 


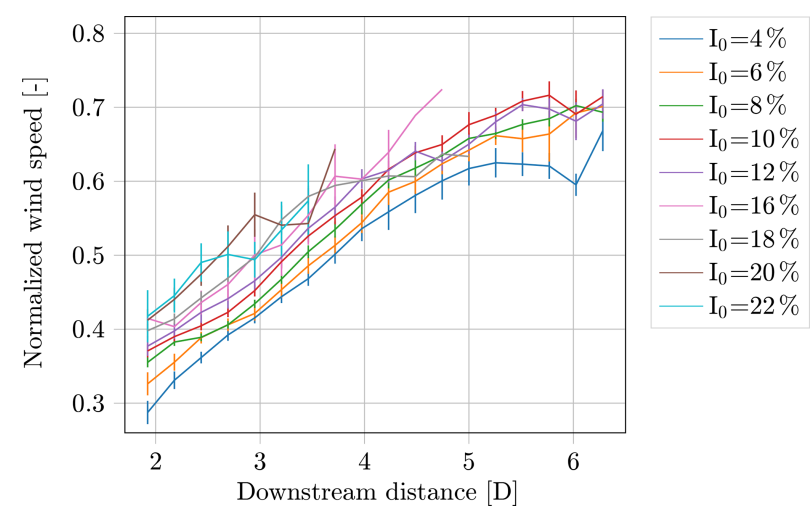

Figure 12. Measured mean value (line) and standard deviation (bar) of the mean value of the minimal wind speed in the HMFR for different turbulence intensity bins with a bin width of $2 \%$.

increasing minimum wind speeds at nearly all downstream distances. Above $10 \%$ turbulence intensity, the case is less clear. Especially at larger downstream distances, the measured normalized minimum wind speed happens to fall below the corresponding lower turbulence intensity bin. An explanation is the reduced number of measurement results in these bins and the higher uncertainty that comes along with it (expressed as error bars). Furthermore, discrepancies in the determined ambient turbulence intensity at the met mast location and the actual turbulence intensity at the wake position could lead to a misinterpretation of the lidar measurements. The farthest distance between the met mast and the location measured by the lidar system that occurs in the analyzed sectors is about $1200 \mathrm{~m}$. With an ambient wind speed of $6.5 \mathrm{~m} \mathrm{~s}^{-1}$, this leads to a wake advection time of $185 \mathrm{~s}$; thus even at the worst conditions, the measured ambient conditions at the met mast should be valid for the measured wakes from the lidar system most of the time. Furthermore, there is no complex terrain at the site, so it can be assumed that the conditions do not change with the wind direction. In addition, the agreement between measurements and simulations is already good in the higher-turbulence-intensity bins. Thus, the recalibration affects only the lower-turbulence-intensity bins with larger amounts of data, while the influence of the calibration on higher turbulence intensities is negligible (see Fig. 13). Therefore, even though there are some discrepancies, the faster recovery of the wind speed deficit due to the higher ambient turbulence intensity can be verified, and the measurements are reliable for the outlined investigation. Thus, it is valid to use these measurement results for comparisons with DWM model simulations and the recalibration of the DWM model in the next section.

\section{Comparison between measurements and DWM model simulation}

Figure 13 compares the measured normalized minimum wind speed in the wake to DWM model simulations. Figure 13a shows results for a relatively low turbulence intensity of $6 \%$, whereas panel (b) contains results for a higher turbulence intensity of $16 \%$. Further results for the remaining turbulence intensity bins are shown in Figs. B1 and B2 in the Appendix. The simulations were carried out for a specific downstream distance, which corresponds to the center of the range gate of the lidar system. It should be noted that the wind speeds measured by the lidar system can be interpreted as a mean value over the whole range gate. However, the wind speed gradient in the axial direction is low and almost linear in the observed downstream distances, so even in the DWM model, the discretization in the downstream direction is $23.4 \mathrm{~m}$ (equivalent to $0.2 \mathrm{D}$ ), which is on the same order as the range gate of $30 \mathrm{~m}$. Therefore, a valid comparison between simulations and measurements is carried out. The wind speed deficit simulations in the HMFR obtained by the DWM model also include the vertical meandering to ensure a correct comparison between measurements and simulations. Three different simulation results with varying definitions of the initial deficit and eddy viscosity description are illustrated. The method called "DWM-Egmond" is based on the definitions of Madsen et al. (2010) and Larsen et al. (2013) and the "DWM-Keck" method is adopted from Keck (2013); see Sect. 6. Figure 13 shows that the DWMEgmond method overestimates the wind speed deficit for all downstream distances and for both turbulence intensities. The simulated minimum wind speed with the DWM-Keck method are in better agreement with the measurement results. This confirms the results in Reinwardt et al. (2018). Especially at higher turbulence intensities (Fig. 13), the results of the DWM-Keck model agree very well with the measurements. For lower turbulence intensities and higher distances (greater than $3 D$ ), there is a relatively large discrepancy between measurements and simulations. A similar observation was made in Larsen et al. (2013) with the model version in Madsen et al. (2010). Aiming at the adjustment of the simulated degradation of the wind speed deficit in Larsen et al. (2013) for cases like the one presented here, the DWM model has been recalibrated and is henceforth called "DWM-Keckc" (see Fig. 13).

The recalibration of the DWM model and accordingly the normalized eddy viscosity definition in the DWM model are based on a least-squares fit of the minimum of the simulated normalized wind speed to the minimum of the measured normalized wind speed for several downstream distances. The definition of the eddy viscosity along with the recalibrated parameters are explained in detail in Sect. 6.3. For the recalibration the measurement results are divided into $2 \%$ turbulence intensity bins. All measurement results from Fig. 12 containing data sets from two different turbines are used for 


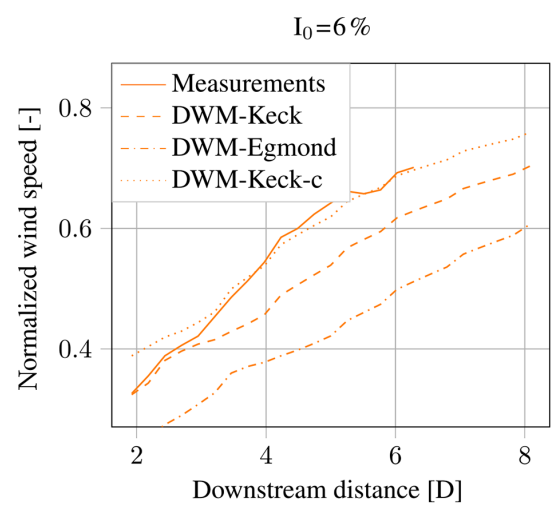

(a)

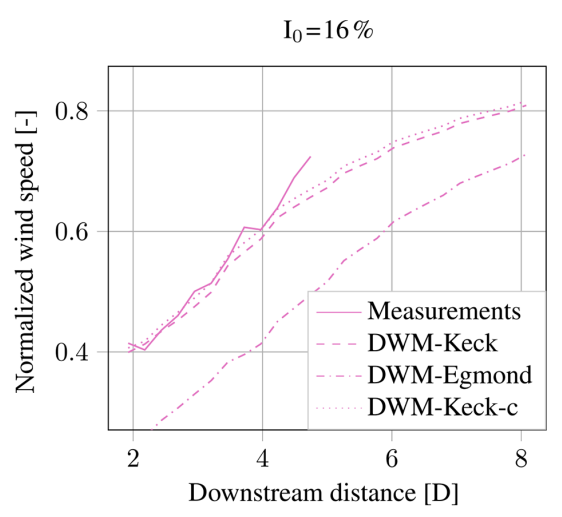

(b)

Figure 13. Comparison of measurements and simulations of the minimum wind speed deficit in the HMFR for different turbulence intensities. The recalibrated model is denoted DWM-Keck-c.

the recalibration. The first turbine is an N117 turbine with $3 \mathrm{MW}$ and the second one is an N117 with 2.4 MW. DWM model simulations were carried out for both turbine types, since the axial induction of both turbines is slightly different under partial load conditions. To calculate a mean value of the simulated minimum wind speed and thus allow a comparison with the results in Fig. 12, simulations with both turbine types are carried out for each turbulence intensity bin and weighted in accordance with the number of measurement results per turbine listed in Table 2. Thus, for example at the ambient turbulence intensity bin of $4 \%$, the mean value of the simulated minimum wind speed consists of the sum of the simulated minimum wind speeds weighted by 0.451 and 0.549 , the weighting factors for WTG1 and WTG2, respectively. Nonetheless, this weighting has only a marginal influence on the overall results, because the axial induction in the considered wind speed range ( 5 to $8 \mathrm{~m} \mathrm{~s}^{-1}$ ) is very similar for these two turbine types (see also thrust and power curves in Fig. 3).

The results of the recalibrated DWM model, denoted Keck-c in Fig. 13, coincide very well with the measurements. In particular, the results for lower turbulence intensities could clearly be improved. For higher turbulence intensities, the influence of the recalibration is less significant and the already good agreement between simulation and measurement results remains unchanged. The same applies to the results in the Appendix in Figs. B1 and B2. Only at the lowest downstream distances and turbulence intensities up to $12 \%$ does the recalibrated model deliver higher deviations than the original model. For downstream distances larger than $3 D$, the recalibrated model leads to more than $10 \%$ lower deviations from the measurements than the original model. For turbulence intensities higher than $16 \%$, the deviation between the recalibrated and original model is smaller than the uncertainties in the measurements; hence no further conclusions about improvements can be made. The uncertainties in accordance with misalignments could be up to $5 \%$ (see also the data fil- tering in Sect. 3). Furthermore, the LOS accuracy of the lidar system itself is about $1.5 \%$ at a wind speed of $6.5 \mathrm{~m} \mathrm{~s}^{-1}$. The root-mean-square error (RMSE) between the measured and simulated normalized minimum wind speed is collected for all analyzed turbulence intensity bins in Fig. 14. A clear improvement of the results due to the recalibrated model version up to an ambient turbulence intensity of $16 \%$ is visible. For higher turbulence intensity bins, the RMSEs of the recalibrated and the original DWM-Keck model version are similar. The DWM-Egmond model delivers significantly higher RMSEs than the other model versions for all turbulence intensity bins. A comparison between the simulated and measured mean wake wind speed over the rotor area has been carried out as well ${ }^{1}$. The improvement of the mean wind speed is less clear in comparison to the normalized minimum wind speed. Yet, there is an improvement or results of equal quality are obtained in almost all turbulence intensity bins. At the tails of the wind speed deficit, the curves are coarse, since fewer scan points are gathered and the influence of turbulence is much higher (see Fig. 9). This leads to an error in the mean wake wind speed but not in the minimum wind speed, which is why the illustration and recalibration of the model are based on the minimum wake wind speed instead of the wake mean wind speed.

Figure 15 compares the final recalibrated DWM model to the original model definition. It shows the minimum normalized wind speed (panel a) and the wind speed at the wake center (panel b) over downstream distances from $0 D$ to $10 D$ for the lower- and the higher-turbulence-intensity cases of $6 \%$ and $16 \%$, respectively. Observing the wind speed at the wake center, higher wind speeds can be seen at lower distances, which derives from the "W" shape of the wind speed at these downstream distances. The comparison of the DWM-Keck model (orange curve) and the recalibrated model DWM-Keck-c (green curve) demonstrates that the re-

\footnotetext{
${ }^{1}$ https://wes.copernicus.org/preprints/wes-2019-89/ access: 17 June 2020).
} 


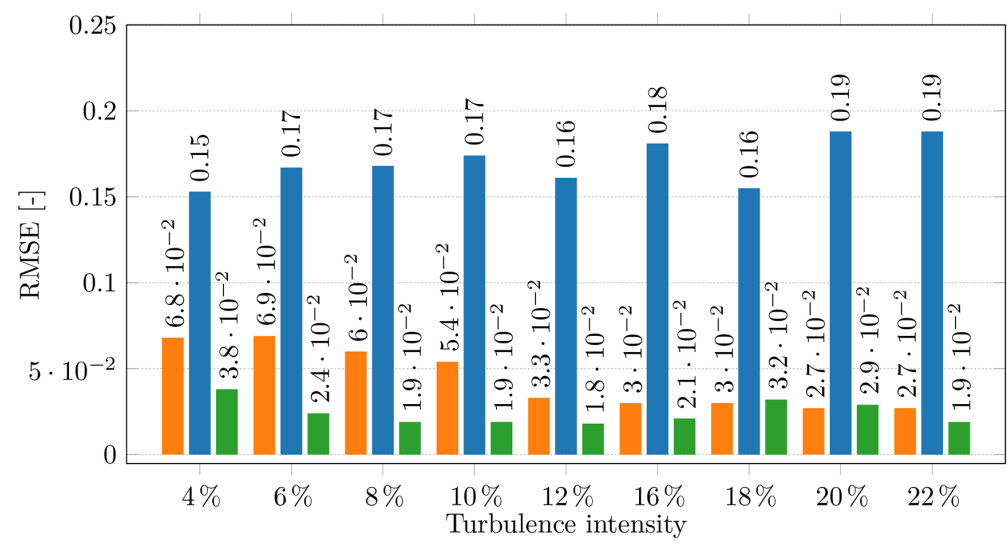

II Keck

II Egmond

I I Keck-c

Figure 14. RMSE between the lidar-measured and the simulated normalized minimum wind speed in the wake.

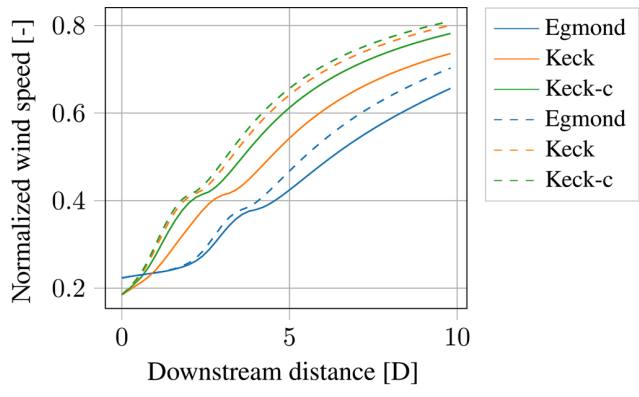

(a)

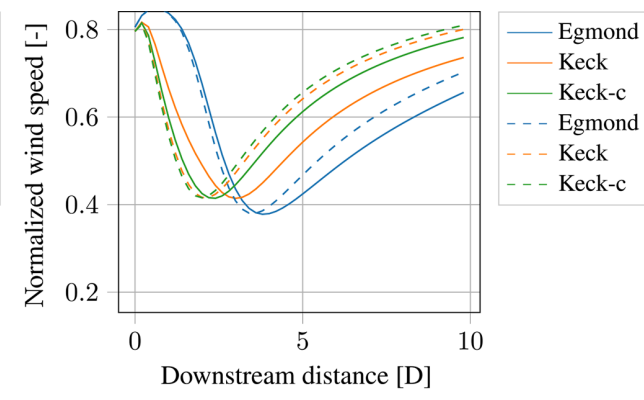

(b)

Figure 15. Simulated minimal normalized wind speed in the MFR (a) and normalized wind speed at the wake center (b) over the downstream distance for a turbulence intensity of $6 \%$ (solid curves) and 16\% (dashed curves). The recalibrated model is denoted DWM-Keck-c.

calibration leads to a shift of the curve towards lower distances. This shift is more pronounced for the lower turbulence intensity, leading to a faster degradation of the wind speed deficit. For the higher turbulence intensity, both curves, orange and green, are very close to each other over all distances. The faster degradation of the wind speed deficit in the recalibrated model version is caused by introducing the function $F_{\mathrm{amb}}$ in the eddy viscosity definition in Eq. (21) as explained in Sect. 6.3. The function increases the eddy viscosity for lower turbulence intensities and thus increases the wind speed deficit degradation in the downstream direction. Contemplating the curve of the minimum wind speed in Fig. 15a, small steps are formed in the curves between $2 D$ and $4 D$ (depending on the used model and the turbulence intensity). These steps correspond to the minimum of the curves in Fig. 15b and are thus related to the transition from the "W" shape of the wind speed deficit towards the Gaussian profile and are consequently caused by the resolution in the downstream direction. These steps were also found in some measurements and could likewise be related to the implied transition zone.

\section{Conclusions}

The study compares measurements of the wind speed deficit with DWM model simulations. The measurement campaign consists of two nacelle-mounted lidar systems in a densely packed onshore wind farm. The lidar measurements were prepared by lidar and wind field simulations to examine whether the scan pattern is suitable for the outlined analysis. Several wind speed deficits that were simultaneously measured at different downstream distances are presented along with their associated meandering time series. The onedimensional scan worked reliably in the field campaign, thus delivering lidar data for a multitude of different ambient conditions. These measurements are compared to the simulated wind speed deficit in the HMFR. The simulation result of the DWM-Keck model is in good agreement, whereas the DWM-Egmond model yields a too low degradation of the wind speed deficit. Furthermore, even the DWM-Keck model shows some discrepancies to the measurements at low turbulence intensities, which is why a recalibrated DWM model was proposed. The recalibrated model improves the correlation with measurements at low turbulence intensities and leads to an agreement at high turbulence intensities, which 
are as good as the original model, thus resulting in a very good overall conformity with the measurements.

Future work will include the analysis of two-dimensional scans as well as measurements with more range gates and higher spatial resolutions. Increasing the number of range gates and scan points will lead to longer scan times, hence preventing further analysis of the wind speed deficit in the MFR and the determination of the meandering time series. Nevertheless, a validation of the wind speed deficit in the FFR with higher resolutions and more distances seems reasonable to also prove the validity of the outlined calibration for further distances. Furthermore, the analyzed models will be assessed in load as well as power production simulations and compared to the particular measurement values from the wind farm. Simulations have shown that the recalibration of the DWM-Keck model can lead to up to $13 \%$ lower loads in the turbulence-dependent components in cases with small turbine distances and low turbulence intensities, whereas for higher turbulence intensities $(>12 \%)$ the difference between the original and the recalibrated DWM-Keck model is less than $5 \%$. The overall influence of the recalibration on the power output is low $(<2 \%$ for all turbulence intensities). So far, only measured single wakes were presented. Yet, a brief analysis demonstrated that multiple wakes can also be recorded with the described measurement setup. A future step will therefore be an analysis of multiple-wake situations. 
Appendix A: Measurement results

Meandering

$\left(\mathrm{U}_{0}=7.98 \mathrm{~m} \mathrm{~s}^{-1}, \mathrm{I}_{0}=0.024, \alpha=0.7, \theta=183.0^{\circ}, \mathrm{d}=2.69 \mathrm{D}\right)$

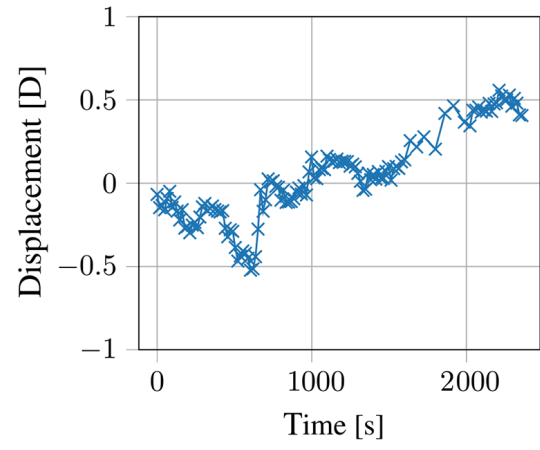

(a)
HMFR

$\left(\mathrm{U}_{0}=7.98 \mathrm{~m} \mathrm{~s}^{-1}, \mathrm{I}_{0}=0.024, \alpha=0.7, \theta=183.0^{\circ}, \mathrm{d}=2.69 \mathrm{D}\right)$

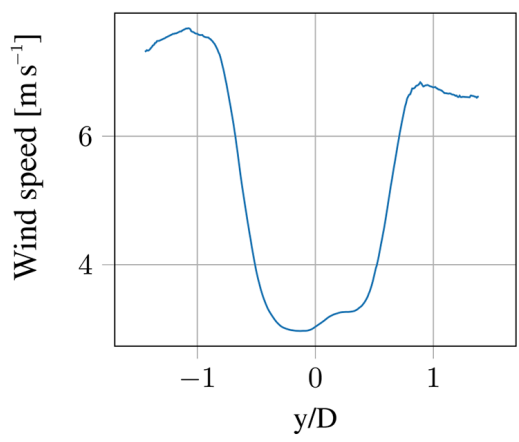

(b)

Figure A1. Meandering time series (a) and wind speed deficit in the HMFR (b) at 2.69D downstream of the turbine. 
Appendix B: Comparison of measurements and DWM model simulation

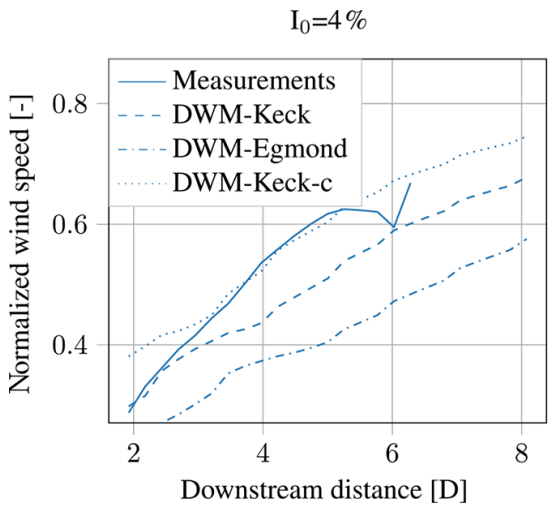

(a)

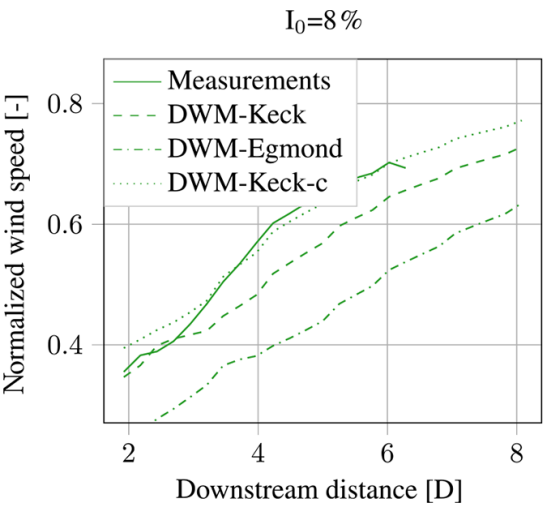

(b)

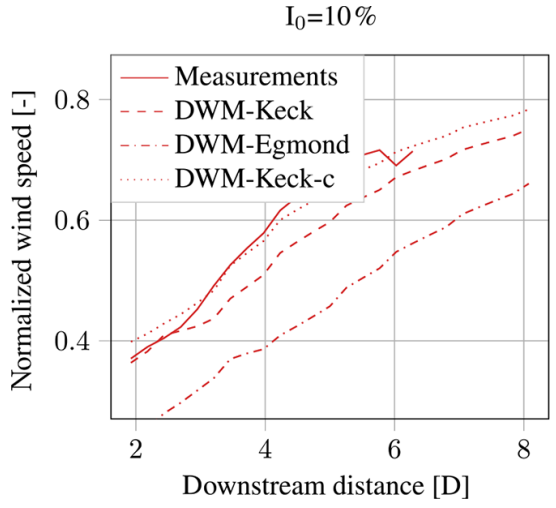

(c)

Figure B1. Comparison of measurements and simulations of the minimum wind speed deficit in the HMFR for different turbulence intensities. The recalibrated model is denoted DWM-Keck-c.

$\mathbf{I}_{0}=12 \%$

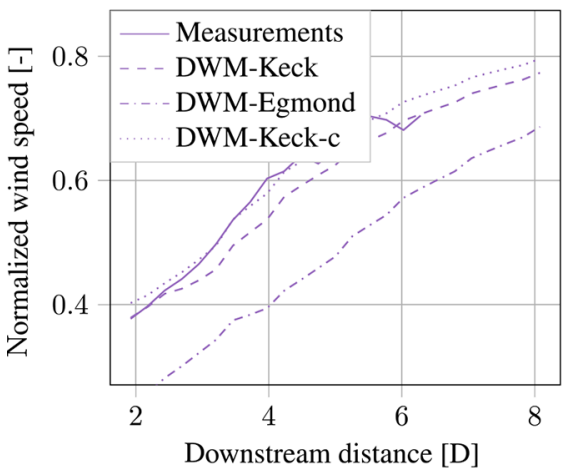

(a)

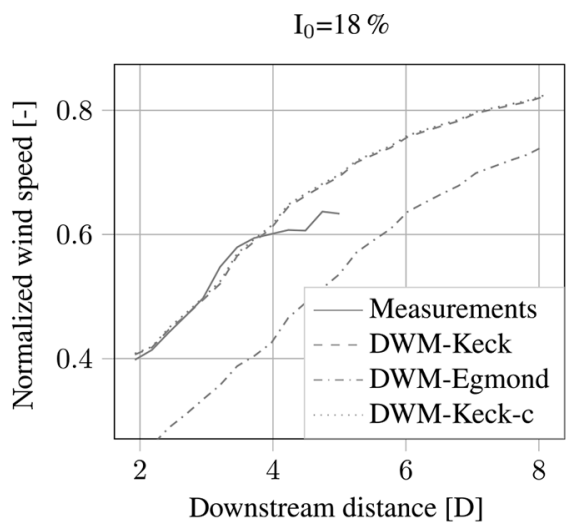

(b)

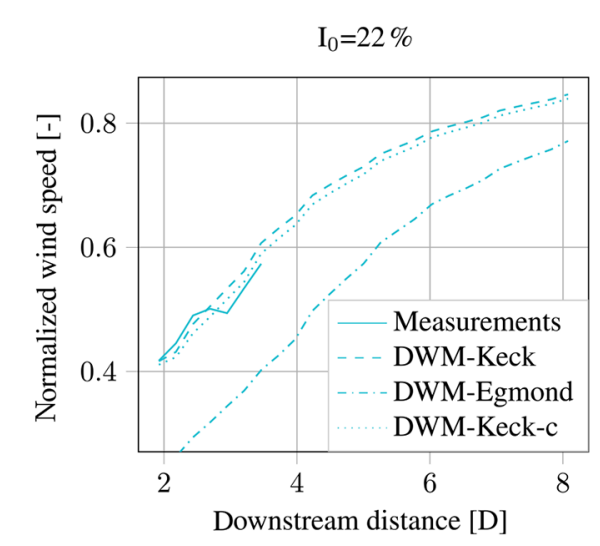

(d)

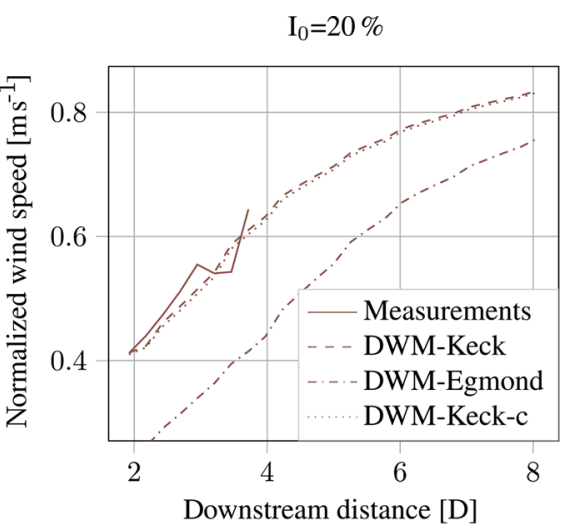

(c)

Figure B2. Comparison of measurements and simulations of the minimum wind speed deficit in the HMFR for different turbulence intensities. The recalibrated model is denoted DWM-Keck-c. 
Code and data availability. Access to lidar and met mast data as well as the source code used for post-processing the data and simulations can be requested from the authors.

Author contributions. IR performed all simulations, postprocessed and analyzed the measurement data, and wrote the paper. LS and DS gave technical advice in regular discussions and reviewed the paper. $\mathrm{PD}$ and $\mathrm{MB}$ reviewed the paper and supervised the investigations.

Competing interests. The authors declare that they have no conflict of interest.

Special issue statement. This article is part of the special issue "Wind Energy Science Conference 2019". It is a result of the Wind Energy Science Conference 2019, Cork, Ireland, 17-20 June 2019.

Acknowledgements. The content of this paper was developed within the project NEW 4.0 (North German Energy Transition 4.0).

Financial support. This research has been supported by the Federal Ministry for Economic Affairs and Energy (BMWI) (grant no. 03SIN400).

Review statement. This paper was edited by Sandrine Aubrun and reviewed by Helge Aagaard Madsen and Vasilis Pettas.

\section{References}

Ainslie, J. F.: Calculating the flowfield in the wake of wind turbines, J. Wind Eng. Ind. Aerodyn., 27, 213-224, 1988.

Bingöl, F., Mann, J., and Larsen, G. C.: Light detection and ranging measurements of wake dynamics Part I: One-dimensional scanning, Wind Energy, 13, 51-61, https://doi.org/10.1002/we.352, 2010.

Frandsen, S.: Turbulence and turbulence-generated structural loading in wind turbine clusters, $\mathrm{PhD}$ thesis, Technical University of Denmark, Roskilde, Denmark, 2007.

Gerke, N., Reinwardt, I., Dalhoff, P., Dehn, M., and Moser, W.: Validation of turbulence models through SCADA data, J. Phys. Conf. Ser., 1037, 072027, https://doi.org/10.1088/17426596/1037/7/072027, 2018.

IEC 61400-1: IEC 61400-1 Ed. 4: Wind energy generation systems - Part 1: Design requirements, Guideline, International Electrotechnical Commission (IEC), Geneva, Switzerland, 2019.

Keck, R.-E.: A consistent turbulence formulation for the dynamic wake meandering model in the atmospheric boundary layer, $\mathrm{PhD}$ thesis, Technical University of Denmark, Lyngby, Denmark, 2013.
Keck, R.-E., de Maré, M., Churchfield, M. J., Lee, S., Larsen, G., and Madsen, H. A.: On atmospheric stability in the dynamic wake meandering model, Wind Energy, 17, 1689-1710, 2014.

Larsen, G. C., Madsen, H. A., Bingöl, F., Mann, J., Ott, S. R., Sørensen, J. N., Okulov, V., Troldborg, N., Nielsen, M., Thomsen, K., Larsen, T. J., and Mikkelsen, R.: Dynamic wake meandering modeling, Tech. Rep. Ris $\varnothing-\mathrm{R}-1607(\mathrm{EN})$, Ris $\varnothing$ National Laboratory, Roskilde, Denmark, 2007.

Larsen, G. C., Madsen, H. A., Larsen, T. J., and Troldborg, N.: Wake modeling and simulation, Tech. Rep. Ris $\varnothing-\mathrm{R}-1653(\mathrm{EN})$, Ris $\varnothing$ National Laboratory for Sustainable Energy, Roskilde, Denmark, 2008a.

Larsen, G. C., Madsen, H. A., Thomsen, K., and Larsen, T. J.: Wake meandering: A pragmatic approach, Wind Energy, 11, 377-395, https://doi.org/10.1002/we.267, 2008b.

Larsen, T. J., Madsen, H. A., Larsen, G. C., and Hansen, K. S.: Validation of the dynamic wake meander model for loads and power production in the Egmond aan Zee wind farm, Wind Energy, 16, 605-624, 2013.

Machefaux, E., Troldborg, N., Larsen, G., Mann, J., and Aagaard Madsen, H.: Experimental and numerical study of wake to wake interaction in wind farms, in: Proceedings of EWEA 2012 - European Wind Energy Conference \& Exhibition, European Wind Energy Association (EWEA), 16-19 April 2012, Copenhagen, Denmark, 2012.

Machefaux, E., Larsen, G. C., Troldborg, N., and Rettenmeier, A.: Single wake meandering, advection and expansion - An analysis using an adapted pulsed lidar and CFD LES-ACL simulations, in: Prodeedings of EWEA 2013-0 European Wind Energy Conference \& Exhibition, European Wind Energy Association (EWEA), 4-7 February 2013, Vienna, Austria, 2013.

Machefaux, E., Larsen, G. C., Troldborg, N., Gaunaa, M., and Rettenmeier, A.: Empirical modeling of single-wake advection and expansion using full-scale pulsed lidar-based measurements, Wind Energy, 18, 2085-2103, https://doi.org/10.1002/we.1805, 2015.

Machefaux, E., Larsen, G. C., Troldborg, N., Hansen, K. S., Angelou, N., Mikkelsen, T., and Mann, J.: Investigation of wake interaction using full-scale lidar measurements and large eddy simulation, Wind Energy, 19, 1535-1551, https://doi.org/10.1002/we.1936, 2016.

Madsen, H. A., Larsen, G. C., Larsen, T. J., Troldborg, N., and Mikkelsen, R.: Calibration and validation of the dynamic wake meandering model for implementation in an aeroelastic code, J. Sol. Energy Eng., 132, 041014, https://doi.org/10.1115/1.4002555, 2010.

Reinwardt, I., Gerke, N., Dalhoff, P., Steudel, D., and Moser, W.: Validation of wind turbine wake models with focus on the dynamic wake meandering model, J. Phys. Conf. Ser., 1037, 072028, https://doi.org/10.1088/1742-6596/1037/7/072028, 2018.

Trujillo, J.-J., Bingöl, F., Larsen, G. C., Mann, J., and Kühn, M.: Light detection and ranging measurements of wake dynamics. Part II: Two-dimensional scanning, Wind Energy, 14, 61-75, https://doi.org/10.1002/we.402, 2011.

Veers, P. S.: Three-Dimensional Wind Simulation, Tech. Rep. SAND88-0152(EN), Sandia National Laboratories, New Mexico, USA, 1988. 